\title{
Les missions populaires de 1840-42 : acteurs principaux et conséquences
}

\section{Louis Rousseau}

Volume 53, 1986

Le renouveau religieux à Montréal au XIX ${ }^{\mathrm{e}}$ siècle

URI : https://id.erudit.org/iderudit/1006967ar

DOI : https://doi.org/10.7202/1006967ar

Aller au sommaire du numéro

\section{Éditeur(s)}

Les Éditions Historia Ecclesiæ Catholicæ Canadensis Inc.

ISSN

0318-6172 (imprimé)

1927-7067 (numérique)

Découvrir la revue

Citer cet article

Rousseau, L. (1986). Les missions populaires de 1840-42 : acteurs principaux et conséquences. Sessions d'étude - Société canadienne d'histoire de l'Église catholique, 53, 7-21. https://doi.org/10.7202/1006967ar
Résumé de l'article

L'échec des soulèvements de 1837-38 signale le point extrême atteint par une crise multidimensionnelle affectant la société bas-canadienne depuis une vingtaine d'années et qui va de pair avec une tiédeur religieuse remarquable. Dès 1839, à l'instigation de $\mathrm{M}^{\mathrm{gr}}$ Lartigue et de $\mathrm{M}^{\mathrm{gr}}$ Bourget, une stratégie pastorale de conversion d'ensemble de la population des paroisses prend forme. Profitant de la présence nord-américaine de $\mathrm{M}^{\mathrm{gr}}$ Forbin-Janson, co-fondateur des Missionnaires de France, une vaste campagne de Missions populaires s'engage dans la région montréalaise à partir de septembre 1840 . Nous analysons ici le rôle des acteurs principaux de ce mouvement : l'initiateur et l'organisateur, $\mathrm{M}^{\mathrm{gr}}$ Bourget; l'importateur de la formule et le prophète, $\mathrm{M}^{\mathrm{gr}}$ Forbin-Janson; les collaborateurs cléricaux, les curés des paroisses touchées et leurs confrères; les sujets de la dramaturgie de conversion, le peuple des paroisses. Durant les deux premières années, près de la moitié du diocèse a été atteinte. La transformation des attitudes et des conduites n'a pas été subite. Mais le mouvement de réveil religieux enclenché, mesuré à la lumière des communions pascales, conduira à la quasi-unanimité à la fin des années 1860 .
Tous droits réservés (C) Les Éditions Historia Ecclesiæ Catholicæ Canadensis Inc., 1986
Ce document est protégé par la loi sur le droit d'auteur. L'utilisation des services d'Érudit (y compris la reproduction) est assujettie à sa politique d'utilisation que vous pouvez consulter en ligne.

https://apropos.erudit.org/fr/usagers/politique-dutilisation/ 


\title{
Les missions populaires de 1840-42: acteurs principaux et conséquences
}

\author{
Louis RousSEAU \\ Département des sciences religieuses \\ Université du Québec à Montréal
}

RÉSUMÉ

L'échec des soulèvements de 1837-38 signale le point extrême atteint par une crise multidimensionnelle affectant la société bas-canadienne depuis une vingtaine d'années et qui va de pair avec une tiédeur religieuse remarquable. Dès 1839, à l'instigation de $M^{g r}$ Lartigue et de $M^{g r}$ Bourget, une stratégie pastorale de conversion d'ensemble de la population des paroisses prend forme. Profitant de la présence nord-américaine de $M^{g r}$ Forbin-Janson, co-fondateur des Missionnaires de France, une vaste campagne de Missions populaires s'engage dans la région montréalaise à partir de septembre 1840. Nous analysons ici le rôle des acteurs principaux de ce mouvement: l'initiateur et l'organisateur, $M^{g r}$ Bourget; l'importateur de la formule et le prophète, $M^{g r}$ Forbin-Janson; les collaborateurs cléricaux, les curés des paroisses touchées et leurs confrères; les sujets de la dramaturgie de conversion, le peuple des paroisses. Durant les deux premières années, près de la moitié du diocèse a été atteinte. La transformation des attitudes et des conduites n'a pas été subite. Mais le mouvement de réveil religieux enclenché, mesuré à la lumière des communions pascales, conduira à la quasi-unanimité à la fin des années 1860.

L'histoire religieuse montréalaise n'est pas un nouveau chantier pour l'historiographie québécoise. On peut sans doute affirmer que les objets qui ont retenu davantage l'attention jusqu'ici sont de nature biographique ou institutionnelle. Peu d'attention a été accordée à la vie religieuse de la masse des fidèles. Or c'est justement dans cette voie que nous nous sommes engagés 
depuis quelques années avec une équipe de l'UQAM $^{1}$ et il serait peut-être utile de présenter quelque chose de notre perspective générale qui donne sens à l'étude des Missions populaires de 1840-42.

Que se passe-t-il donc dans cet immense espace humain situé dans le triangle sud-ouest du Québec au milieu du XIX ${ }^{\mathrm{e}}$ siècle ?2 Pour dire les choses rapidement, nous devons parler d'un processus général de transformation qui dans l'espace de quelques décennies a vu la population engagée dans une suite de crises de nature démographique, économique, sociale, politique et religieuse, d'amplitude exceptionnelle, qụi ont semblé conduire à l'impasse des soulèvements de 1837-38. Mais les années quarante et cinquante voient renaître d'une façon surprenante les diverses facettes de la vie de telle sorte que l'on peut sans doute parler, avec l'anthropologue Wallace (1956), d'un véritable processus de "revitalisation" de cette société. Les transformations de l'économie montréalaise comme celles des rapports sociaux vont maintenant s'ajuster à l'intérieur d'une nouvelle image de soi et du monde dont se dote la population canadienne catholique à la faveur d'un réveil religieux inauguré à l'occasion des campagnes de Missions populaires, diffusé ensuite dans les conduites par le biais des mouvements de Tempérance et des confréries pieuses, le tout activé par et activant une élite militante cléricale, religieuse et laïque, ce qui aura pour effet de placer la religion au centre de l'univers mental et les responsables d'Église au centre des opérations de contrôle et de régulation caractéristiques de cette nouvelle société. Voilà, trop vite résumée, l'image de vastes changements multidimensionnels qui oriente toute notre recherche actuelle. On pourrait employer d'autres termes comme ceux de "réforme» ou de «contre-réforme catholique» ou encore de "réaction catholique», mais il me semble plus fécond d'embrasser un phénomène de durée et de profondeur beaucoup plus étendu qui, entre 1820 et 1880 , a vu toute une société douter graduellement de plus en plus de la qualité de l'ensemble de ses savoir-faire, y compris de ses ressources religieuses, élaborer de nouvelles solutions qui ont semblé mener à l'échec collectif de 1837-38, puis se rebâtir une cohérence en allant puiser des éléments choisis dans sa tradition catholique (d'où l'expression technique de «réveil») et retrouver ainsi une dynamique collective capable de résoudre

1 L'équipe du projet Renouveau religieux montréalais au XIXe siècle est dirigée par Louis Rousseau (sciences religieuses) en collaboration avec Frank W. Remiggi (géographie). Francine Girard-Ducasse agit à titre d'assistante principale et Jean-Patrice Arès, Catherine Cordeau ainsi que Céline Payette œuvrent comme assistants. Ces deux dernières ont collaboré plus particulièrement à la préparation de cet article. Le projet bénéficie de l'appui financier de l'UQUAM, du CRSHC et du FCAR.

2 Une première description géographique du territoire étudié a été réalisée par Frank W. Remiggi, "Esquisse géographique du territoire diocésain de Montréal, 1820-1852», dans R. Litalien, éd., L'Église de Montréal, 1836-1986, Montréal, Fides, 1986, p. 30-39. 
d'une façon satisfaisante (je ne dis pas optimale) les problèmes nouveaux posés par la situation.

Comme j'ai eu l'occasion de présenter plus en détail la perspective de notre recherche ainsi que certains de ses premiers résultats concernant, entre autres, la campagne de prédication populaire de $1840-42^{3}$, je ne retiendrai ici que deux questions principales: quel a été le rôle des différents groupes impliqués dans les Missions pour le peuple et quel a été l'impact à court et à moyen terme de ces campagnes sur le réveil religieux des populations de la grande région montréalaise?

\section{Les acteurs principaux}

À la différence de plusieurs mouvements de revitalisation globale de sociétés généralement de taille plus restreinte que nous décrit la littérature ethnographique, nous ne retrouvons pas, à l'origine du réveil religieux montréalais, une personnalité prophétique faisant l'expérience d'une illumination intérieure nouvelle qu'elle cherchera ensuite à faire partager par l'ensemble de son groupe. Nous sommes en présence d'un ensemble d'acteurs sociaux qui innovent dans l'action, mais à partir des places et selon des rôles rendus possibles par une appartenance institutionnelle préexistante. Nous pouvons isoler ainsi, pour l'analyse, quatre fonctions principales: l'initiateur, $\mathrm{M}^{\mathrm{gr}}$ Bourget; le prophète, $\mathrm{M}^{\mathrm{gr}}$ de Forbin-Janson; les collaborateurs cléricaux, une équipe de prêtres diocésains bientôt relayés par les premiers Oblats (décembre 1841); et finalement le peuple des paroisses qui, on l'oublie trop souvent, avait un rôle essentiel à jouer dans la dramatique de la conversion qui allait s'engager sur tout le territoire. Décrivons à grands traits le comportement de ces différents agents au cours des «Missions et des Retraites pour le peuple»4.

\section{L'initiateur: $M^{g r}$ Bourget}

Il ne fait aucun doute que l'initiateur des Missions pour le peuple a été Ignace Bourget. $\mathrm{M}^{\mathrm{gr}}$ Lartigue et lui-même connaissaient sans doute par les journaux et les revues reçus de France l'Oeuvre des Missionnaires de France fondée par Forbin-Janson et Eugène de Mazenod dans la France de la

3 "À l'origine d'une société maintenant perdue: le réveil religieux montréalais de 1840 ", dans Y. Desrosiers, éd., Religion et culture au Québec. Figures contemporaines du Sacré, Montréal, Fides, 1986, p. 71-92.

4 L'expression est de Bourget dans sa circulaire du 9 septembre 1840 envoyée à son clergé avant la retraite de Terrebonne. Mandements, circulaires, etc., des évêques du diocèse de Montréal, vol. I, p. 89. Les termes de Mission et de Retraite sont considérés comme équivalents à l'époque, mais il faut se garder de confondre la mission traditionnelle d'un prêtre allant visiter les fidèles d'une région n'ayant pas encore de desservant régulier avec l'activité concertée dont il va être question ici. 
Restauration. L'idée d'établir dans le diocèse cette pratique de conversion des masses aurait pris corps au cours de l'automne 1839 lorsqu'on apprit la présence à New York de Forbin-Janson. Une invitation lui fut sans doute envoyée dès ce moment et les prêtres réunis pour la première retraite sacerdotale de l'Église canadienne prièrent ensemble pour que la réponse $\mathrm{du}$ fougueux évêque exilé fut favorable 5 . S'il n'eut pas la paternité de l'idée, Bourget fut entièrement responsable de sa mise en œuvre, à commencer par le travail de convaincre son clergé de l'opportunité d'une pareille initiative qui dérangerait bien des habitudes 6 .

Si l'objectif d'une campagne de Missions populaires était clair (et impliquait dès le départ l'implantation à court terme d'un groupe d'hommes spécialisés qui «retraceront $[. .$.$] la vie apostolique»), la planification dut se$ faire en souplesse, car l'évêque de Montréal avait tout à apprendre et ne disposait pas à sa guise de son principal acteur, Mgr de Forbin-Janson, ni de ses curés.

Dans un premier temps, il fut décidé de choisir des paroisses centrales "afin que les paroisses environnantes en profitent». Quelques mois plus tard, avec la possibilité de multiplier les missions, s'imposa la politique d'une seule paroisse à la fois. Mais la demande devait en être faite par les pasteurs locaux. Le curé de Terrebonne fut le premier à se porter volontaire en septembre 1840. M. Quiblier, de Notre-Dame de Montréal, M. Crevier, de Saint-Hyacinthe, M. St-Germain, de Saint-Laurent, puis M. Bonin, de SainteScholastique, permirent au projet de Bourget de commencer à prendre chair à l'automne et à l'hiver 1840-41.

Le rôle de l'évêque de Montréal est à chaque fois un peu le même. Il constitue l'équipe de prêtres qui seconderont le prédicateur principal pour les confessions très nombreuses et souvent compliquées et les différentes cérémonies faisant partie du rituel de la retraite. Il leur donne le pouvoir d'absoudre un certain nombre de cas normalement réservés à l'évêque ou au Pape (questions reliées au territoire des interdits sexuels, pour l'essentiel). Il rédige dans la plupart des cas une lettre pastorale annonçant les exercices de la mission, faisant valoir ses avantages et exhortant les paroissiens à se préparer à de grands changements de conduite par des exercices de pénitence

5 C'est le scénario le plus vraisemblable que permet de reconstituer la Circulaire de Bourget, ibid.

6 Tous n'étaient pas enthousiastes au départ. Même après le succès de la Mission de Terrebonne et surtout celui de Notre-Dame, beaucoup doutaient encore de son opportunité. En témoigne cette confidence du curé de Sainte-Scholastique à la veille de la mission dans sa paroisse: "L'ennemi du bien a mis dans la tête de plusieurs de mes voisins que c'est une mauvaise démarche.» Bonin à Bourget, Sainte-Scholastique, 15 janvier 1841, Archives des Jésuites du Canada français (ASJCF), dossier Sainte-Scholastique. 
et la prière. Il donne des consignes précises au curé concernant l'aménagement de l'église, le chant, les sonneries de cloches, la pompe exceptionnelle des cérémonies, les objets comme les cierges et les médailles et même le style de la cuisine sacerdotale qui doit être plus sévère qu'à l'accoutumée.

Outre son rôle d'initiateur, de planificateur et de metteur en scène, Bourget assume à au moins trois reprises celui de prédicateur principal, prolongeant ainsi ses exhortations écrites par sa parole vive (Contrecœur et Berthierville, en février 1841; Boucherville, en février 1842). En somme, c'est grâce à lui que nous pouvons parler de campagne concertée visant la conversion des masses et menée à un train que nous n'oserions dire «d'enfer» compte tenu du contexte, mais qui va rapidement taxer au maximum les ressources sacerdotales disponibles.

\section{Le prophète modèle: $M^{g r}$ de Forbin-Janson}

Le jeune évêque de Montréal savait prendre l'initiative et organiser au fur et à mesure le déroulement d'une activité concertée et de grande envergure. Mais il ignorait presque tout du déroulement concret d'une Mission populaire. Il fallait disposer d'un modèle et, si possible, d'un prophète venant déclencher, par son message, une réorganisation efficace des représentations, des attitudes et des conduites religieuses. Cela devait être le rôle de $M^{\mathrm{gr}} \mathrm{de}$ Forbin-Janson en qui, au surplus, Bourget allait trouver un autre père ${ }^{7}$.

Un pontife étranger que sa main nous envoie,

Apparaît parmi nous comme un ange du ciel,

Pour abattre le vice et conduire à la voie

L'infidèle brebis du bercail d'Israël.

Sa voix, sa voix d'apôtre, éloquente et sublime,

À nos yeux déroulant ses terribles tableaux,

Y faisait entrevoir, à nos âmes l'abîme

À nos corps l'horreur des tombeaux ${ }^{8}$.

N.-E. Dionne (1910) ${ }^{9}$, F.-X. Côté (1941-42) ${ }^{10}$, plus récemment Claude Galarneau (1985) ${ }^{11}$, ont décrit la campagne nord-américaine et

7 «Et moi, que ferai-je pour entretenir ce doux commerce d'amitié et ces tendres liaisons qui m'ont fait éprouver tant de bonheur? Où irai-je pour vous rencontrer chacun des jours que nous serons séparés l'un de l'autre?» Bourget à Mgr de Nancy, Montréal, 19 nov. 1841, Archives de la Chancellerie de l'Archevêché de Montréal (ACAM), R.L., 2, 410.

8 "L'évêque de Nancy", dans James Huston, Répertoire national, Montréal, édité par Robert Melançon, VLB éd., 1982, t. II, p. 202.

9 N.-E. Dionne, Mgr de Forbin-Janson, évêque de Nancy. Sa vie, son œuvre au Canada, Québec, Laflamme, 1910.

10 F.-X. Côté, «Mgr de Forbin-Janson, évêque de Nancy et de Toul et le mouvement du Québec vers 1840", Rapport de la Société canadienne d'histoire de l'Église catholique, vol. 9 (1941-1942), p. 95-118.

11 Claude Galarneau, «Mgr Forbin-Janson au Québec en 1840-1841 », dans Jean Hamelin et Nive Voisine, éds, Les Ultramontains canadiens-français. Études d'histoire religieuse présentées en hommage au professeur Philippe Sylvain, Montréal, Boréal Express, 1985, p. 121-142. 
québécoise de l'évêque de Nancy. Il n'y a pas lieu de résumer ici l'excellente synthèse de Galarneau puisque nous nous limitons à la mise en lumière des fonctions spécifiques aux différents acteurs au cours des campagnes missionnaires de 1840-42.

Le fondateur des Missionnaires de France a importé une formule pastorale inédite qu'il avait eu le temps d'expérimenter et de mettre bien au point durant une quinzaine d'années dans différentes provinces culturelles de la France. Son originalité ne tient pas tellement à la présentation éloquente d'une suite de thèmes de prédication visant la conversion du pécheur. Les exercices de la neuvaine de saint François-Xavier tenus à Notre-Dame de Montréal depuis plusieurs années, par exemple, utilisaient une suite identique de thèmes dont les trois premiers quarts étaient consacrés aux "vérités terribles de la religion» pour déboucher ensuite sur la présentation des "vérités consolantes" pour les croyants. La nouveauté réside plutôt dans la mise en œuvre de toute une dramatique créant une rupture dans le temps habituel pour faire en sorte que les paroissiens prennent une conscience viscérale de l'horrible condition de pécheurs dans laquelle ils se trouvent et qui explique aussi bien les malheurs et le chaos qu'ils expérimentent présentement qu'elle rend inévitable leur échec dans l'éternité. Différentes mises en scène rituelles sont utilisées pour provoquer cette prise de conscience de la véritable identité de chacun. Au fur et à mesure qu'elle se produit, le processus de transformation positive s'enclenche par le moyen du traitement spécialisé de la confession pour lequel on a prévu une équipe nombreuse de confesseurs pourvus des pouvoirs nécessaires à l'absolution des cas les plus compliqués. Théoriquement la Mission devait durer tant que tous les paroissiens n'étaient pas entrés personnellement dans le drame de la conversion.

Forbin-Janson aura d'abord transmis ce savoir-faire à ses collaborateurs montréalais, à commencer par Bourget lui-même. Cette forme de pastorale de la conversion dont des communautés sacerdotales feront ensuite leur spécialité caractérisera la vie paroissiale catholique par la suite, alors même que l'unanimité de la pratique se sera chez nous installée à demeure, lui faisant perdre de ce fait une grande partie de sa fonction primitive dans le réveil religieux ${ }^{12}$.

Mais l'ancien évêque de Nancy a fait beaucoup plus que d'importer une nouvelle formule d'intervention pastorale. Il a joué d'abord et avant tout le

12 Voir J.-P. Dolan, Catholic Revivalism: The American Experience, Notre Dame, University Press, 1978. 
rôle de porte-parole principal du message d'origine divine adressé à la population des paroisses ${ }^{13}$.

En 14 mois il a été entendu par les fidèles d'au moins 21 paroisses, soit la moitié de celles qui ont été touchées par les Missions. Et si nous savons lire les documents qui nous laissent entrevoir quelle image s'était construite autour de lui à l'époque, il devient évident qu'on se le représentait comme un personnage religieux exceptionnel. «Pontife étranger», «ange du ciel», écrit le poète anonyme, "dirigé vers nous (par) la Divine Providence», écrit Bourget dans sa circulaire initiale à son clergé, ce ne sont là que des exemples de l'espèce d'aura qui entourait son activité de prophète et messager du TrèsHaut. Il n'était pas d'ici, il avait posé ses pieds sur les traces mêmes des pas de Jésus en Palestine, il avait échappé presque miraculeusement à la noyade dans les eaux froides du port de Montréal, les gens simples touchaient à ses vêtements espérant en recevoir une énergie bénéfique, sa parole, depuis la première retraite de Québec, suscitait partout l'enthousiasme des foules: voilà autant d'indices qui nous autorisent sans doute à reconnaître l'image populaire d'un prophète dont le message s'impose avec autorité.

Et ce message obéissait à l'argumentation prophétique traditionnelle bien connue de tous. Les malheurs dont chacun faisait l'expérience n'étaient que la conséquence des années d'égarement antérieures. La conversion permettrait de se retrouver sous la protection efficace de Dieu qui ne manquerait pas de restaurer la prospérité individuelle et nationale et d'assurer le salut éternel des âmes.

L'éloquence de l'homme facilement appréciable par une population dont c'était le mode de communication le plus valorisé, son charisme personnel, l'image qui se répandit de lui par les journaux et le bouche-àoreille, voilà le déclencheur qu'il fallait pour qu'une population d'où semblait absente antérieurement toute ferveur religieuse, vibre au son de l'appel prophétique à la conversion et envahisse les confessionaux afin de transformer sa vie.

\section{Lès collaborateurs cléricaux}

Si certains preachers évangéliques contemporains peuvent évaluer le succès de leur action en comptant ceux qui se sont levés dans l'assemblée pour annoncer publiquement leur prise de position en faveur de Jésus, les prédicateurs catholiques du $\mathrm{XIX}^{\mathrm{e}}$ siècle pouvaient faire de même en comptant le nombre de personnes confessées. Et pour cela il fallait prévoir

13 Bourget avait également remis ses pleins pouvoirs épiscopaux à l'évêque de Nancy en le nommant son Grand Vicaire pour la durée de la campagne de Mission. Il disposait ainsi de la pleine autorité canonique pour résoudre sur le champ tous les problèmes. ACAM, RL, 2, f. 163, 2 septembre 1840 . 
une équipe sacerdotale assez nombreuse et disposant des facultés canoniques nécessaires pour absoudre sans délais inutiles tous les cas susceptibles de se présenter. D'où la participation d'un troisième groupe d'acteurs dont la tâche principale serait d'entendre les confessions.

Fascinée par les figures principales que sont Forbin-Janson et Bourget, l'historiographie des Missions populaires ne s'est pas intéressée jusqu'ici au groupe des collaborateurs cléricaux sans lesquels, il faut bien le dire, cette campagne n'aurait pu être réalisée avec profit.

Nous avons déjà évoqué le rôle des curés. L'initiative de la tenue d'une retraite dans leur paroisse leur appartenait. Lorsqu'en accord avec $\mathrm{M}^{\mathrm{gr}}$ Bourget le moment en avait été fixé, ils devaient préparer leurs fidèles à être dans de bonnes dispositions et veiller à l'organisation matérielle de l'événement, suivant les directives de Forbin-Janson lorsqu'il était présent, ce qui n'était pas une mince besogne compte tenu des exigences exceptionnelles des rituels solennels, de l'afflux des fidèles et de l'arrivée d'une équipe sacerdotale nombreuse à loger et à nourrir durant plusieurs jours. Ils avaient, enfin, la responsabilité exclusive d'assurer le suivi de cet événement de conversion, à tous égards la partie la plus difficile de leur responsabilité et celle pour laquelle il n'existait aucune préparation.

Le travail du curé et du prédicateur principal était secondé par un groupe de prêtres venus de l'extérieur en nombre suffisant pour faire face à la lourde besogne. Pour la retraite de Terrebonne (oct. 1840), une paroisse ne comptant probablement pas plus de 1000 personnes en âge de communier, Bourget fit accompagner Forbin-Janson d'un secrétaire et de huit autres prêtres. Pour celle de Notre-Dame (approximativement $16000 \mathrm{commu}-$ niants), il offrit à $\mathrm{M}$. Quiblier 17 curés de la région et nous savons qu'au moins sept d'entre eux se joignirent aux prêtres du Séminaire pour le mois que dura la Mission. Au moins cinq prêtres s'adjoignirent au curé de SaintLaurent pour sa mission qui regroupait trois paroisses environnantes. Pour Sainte-Scholastique, en janvier 1841, le curé Bonin qui prévoyait la présence de 10 à 12 mille personnes sollicita l'intervention de 10 à 12 confesseurs. Et ainsi de suite. Que l'on ait réussi à regrouper un aussi grand nombre de curés de campagne pour des durées assez longues et obtenu la collaboration de leurs voisins pour les remplacer en «binant» le dimanche, à une époque de disette sacerdotale, marque bien l'importance accordée à la campagne des Missions par Mgr Bourget et une partie significative de son clergé. Cela nous donne également une certaine mesure de la tâche qui attendait ces collaborateurs. Ceux-ci constituaient un chœur liturgique imposant lorsqu'ils officiaient au cours des rituels préparés avec minutie par le secrétaire. S'il ne faut jamais sous-estimer l'importance de la dimension théâtrale des retraites, il reste que l'occupation essentielle des collaborateurs sacerdotaux tenait à la confession.

La retraite devait amener chacun à gémir de ses péchés et à les confesser de manière à en obtenir l'absolution durant le déroulement même de la 
Mission. Dans bien des cas, les pénitents étaient amenés à révéler des situations cachées depuis longtemps et dont l'absolution échappait au pouvoir normal du curé de la paroisse. L'homme qui se repentait d'avoir connu charnellement une sœur de sa femme avant de se marier, par exemple, ne pouvait que s'entendre interdire toute relation conjugale future à moins d'obtenir une dispense de premier degré d'affinité. Cela risquait fort de le décourager. Aussi avait-on obtenu de Rome, dès mai 1840, un indult permettant d'accorder sur le champ toutes les dispenses requises suite à la violation d'un certain nombre d'interdits sexuels.

Chaque prêtre collaborateur à une Mission disposait donc des pouvoirs d'archiprêtre diocésain et des facultés très spéciales octroyées par l'indult romain du 31 mai $1840^{14}$. Il pouvait par conséquent octroyer certaines dispenses, restaurer des mariages contractés malgré des empêchements, procéder secrètement à des remariages rendus nécessaires, bénir et indulgencier divers objets sacrés et, puisque le travail de la confession risquait fort de le tenir occupé du matin jusqu'au soir sans discontinuer, il pouvait déplacer le moment de son bréviaire à volonté ${ }^{15}$.

Nous ne connaissons malheureusement pas systématiquement les noms de ces précieux collaborateurs. St-Germain, Porlier, Th. Durocher et Caron participèrent à trois des quatre premières retraites; d'autres à une seule. Mais il était impossible de constituer un corps même semi-permanent de prêtres diocésains missionnaires. Après un an de campagnes, Bourget avouera que son clergé est complètement exténué et doit se reposer. L'arrivée des Oblats en décembre 1841 allait marquer l'établissement régulier des Missions et des Retraites pour le peuple et clore la phase initiale qui avait taxé au maximum les ressources assez maigres du clergé diocésain.

\section{Le peuple}

Il est temps de passer au dernier groupe impliqué dans les missions populaires, le peuple lui-même, la masse des fidèles des paroisses. La recherche à venir devra leur accorder une attention plus grande, car ils ne sont pas les récepteurs passifs d'un message dogmatique. La Retraite leur donne le rôle essentiel dans la performance collective. L'écoute du scénario du salut de l'humanité par la Croix n'a pour but que de les faire entrer dans l'action: se reconnaître pécheurs, souffrir de cette condition au point de vouloir la changer, demander l'aide de la Puissance divine et la recevoir ensuite en se confessant et en communiant. La rhétorique de persuasion mise

14 Cette date est le meilleur indice trouvé à ce jour permettant d'affirmer que la préparation effective de la campagne de Missions populaires a cornmencé sous l'épiscopat de Lartigue, bien avant l'arrivée soudaine de Forbin-Janson à Québec en septembre 1840 .

15 Bourget à Forbin-Janson, Montréal, 5 septembre 1840, ACAM, R.L. 2, f. 218. 
en œuvre par le clergé devait déboucher sur une pragmatique de la transformation complète de la vie. Écoutons Bourget énoncer le programme:

...les grandes vérités de la religion, qui ne font que peu d'impression dans d'autres temps, remuent toutes les puissances de l'âme dans la retraite. Alors cette Âme désabusée de tout ce qui l'avait charmée, se livre aux gémissements à la vue de tant de désordres qu'elle a à se reprocher. Tant de fornications, d'adultères, d'impuretés de toute espèce qui font rougir la nature, tant d'injustices, de vols et de [rapines], tant d'excès d'intempérance et d'ivrognerie, tant de blasphèmes et d'imprécations, tant de confessions nulles, tant de communions sacrilèges, tant d'indifférence pour les devoirs les plus sacrés de la religion; une vie presqu'entière passée sans pénitence, sans jeünes, sans abstinence, sans messe, sans confession, sans communion: voilà ce qui plonge l'âme pénitente dans un abyme de douleur; et ce qui la pénètre des sentiments d'une parfaite contrition. Oh! vous verrez vousmêmes N.T.C.F... Les torrents de larmes qui arroseront les confessionnaux pendant ces jours de salut prouveront la vérité de ce que nous vous disons. ${ }^{16}$

Il n'y avait donc que peu de spectateurs au cours de ces retraites (quelques protestants à l'occasion, dont le gouverneur anglais venu entendre Forbin-Janson à Notre-Dame), mais une foule participante assez rapidement prête, semble-t-il, à entrer dans l'action intime annoncée par son évêque et créant ainsi par son propre mouvement une incitation supplémentaire à la transformation des plus réticents.

Le temps et les études précises nous manquent pour pousser plus avant dans la description des comportements de la foule durant la Mission. Il faut tâcher d'examiner maintenant l'impact de ces Retraites populaires sur les conduites religieuses des catholiques de la grande région montréalaise.

\section{L'impact des missions sur les conduites religieuses}

À la lecture des reportages publiés au cours des Missions populaires par les Mélanges religieux édités par les prêtres de l'évêché, l'on est porté à conclure au succès total et instantané de la campagne entre 1840 et 1842: présence massive des fidèles aux exercices, enthousiasme universel suscité par l'éloquence de Mgr Forbin-Janson, invasion des confessionnaux, création de sociétés de tempérance, érection de croix commémoratives ou autres monuments, la majorité des paroisses touchées par le blitz pastoral concerté. L'objectif de renouveler rapidement la ferveur des fidèles semblerait avoir été atteint. Qu'en est-il au juste?

La réponse à cette question n'est pas aussi facile. Il y a tout d'abord le problème de la sélection des indicateurs pertinents. Le nombre des présences compte, bien sûr. Mais comment apprécier la transformation des mentalités

16 Lettre Pastorale de Mgr l'évêque de Montréal aux habitants de la paroisse de Saint-Louis de Terrebonne pour leur annoncer une retraite spirituelle. Montréal, 5 septembre 1840 . ACAM, Pièces et Actes, t. 3, f. 164r. 
et des conduites? À cet égard la correspondance des pasteurs devrait être consultée en priorité, car le réveil religieux auquel ils travaillaient devait rapprocher le comportement de leurs fidèles de la norme ecclésiale contemporaine dont ils assuraient le contrôle. Nous n'avons pas encore dépouillé systématiquement la correspondance en provenance des curés. Mais jusqu'ici nous avons été déçu: très peu de descriptions des événements, encore moins de commentaires sur les effets à court terme de la Mission sur leur territoire. Avant d'examiner ce que peuvent nous révéler certains indicateurs quantitatifs tels celui de la conduite face au devoir pascal, commençons par prendre la mesure de la population atteinte durant les deux premières années.

Entre octobre 1840 (Terrebonne) et septembre 1842 (Saint-Valentin) au moins 42 paroisses parmi les 105 existantes ont été touchées par la Mission (40\%). Nous estimons que 100000 personnes ont été ainsi mises en situation de Retraite plus ou moins intense selon la durée des exercices qu'il est souvent impossible de connaître. Plus de la moitié du diocèse restait à atteindre, mais on peut sûrement parler d'un mouvement déclencheur de forte densité. La carte que nous en avons dressée permet d'identifier quelques zones de concentration et certains espaces plus négligés. Le nord-ouest du diocèse a été privilégié, de même que le centre montréalais et la côte sud de Montréal, alors que les zones qui deviendront plus tard les diocèses de Joliette et de Valleyfield n'ont à peu près pas été touchées. Nous n'avons pu encore relier avec certitude les zones d'intervention à des facteurs explicatifs précis comme la tiédeur religieuse, l'état des mœurs, l'activité des nouveaux prédicants protestants ou le dynamisme pastoral du clergé local ${ }^{17}$. Il est probable que Bourget ait préféré les cas difficiles. La pauvreté des données avant 1839 freine l'explication des choix pastoraux en même temps qu'elle interdit le plus souvent la mesure quantitative des transformations.

Quelques bribes de correspondance permettent quand même de tracer quelques esquisses partielles des différents types de résultats à court terme. À Terrebonne, 15 mois après la retraite, les paroissiens semblent avoir adopté une conduite plus ascétique puisque la majorité d'entre eux s'est abstenue d'un grand bal donné au village, méritant ainsi d'être proposée en modèle à tout le diocèse ${ }^{18}$. À Notre-Dame de Montréal, l'évêque de Nancy «a donné un bon coup de seine: beaucoup de petits poissons ont été pris, mais très peu

17 Nous possédons des informations sur les taux de pascalisants avant 1840 dans le cas de quatre paroisses situées dans la zone privilégiée et à chaque fois la situation antérieure aux Missions semble avoir été catastrophique: Sainte-Scholastique (32\% en 1838), Notre-Dame (42\% en 1840), Saint-Augustin (42\% en 1839), Saint-Benoît (46\% en 1839). Le territoire visé ne l'a vraisemblablement pas été au hasard.

18 Bourget au curé Porlier, Montréal, 4 février 1842, ACAM, R.L., 2, p. 488. 


\section{PAROISSES MISSIONNÉES, 1840-1842}

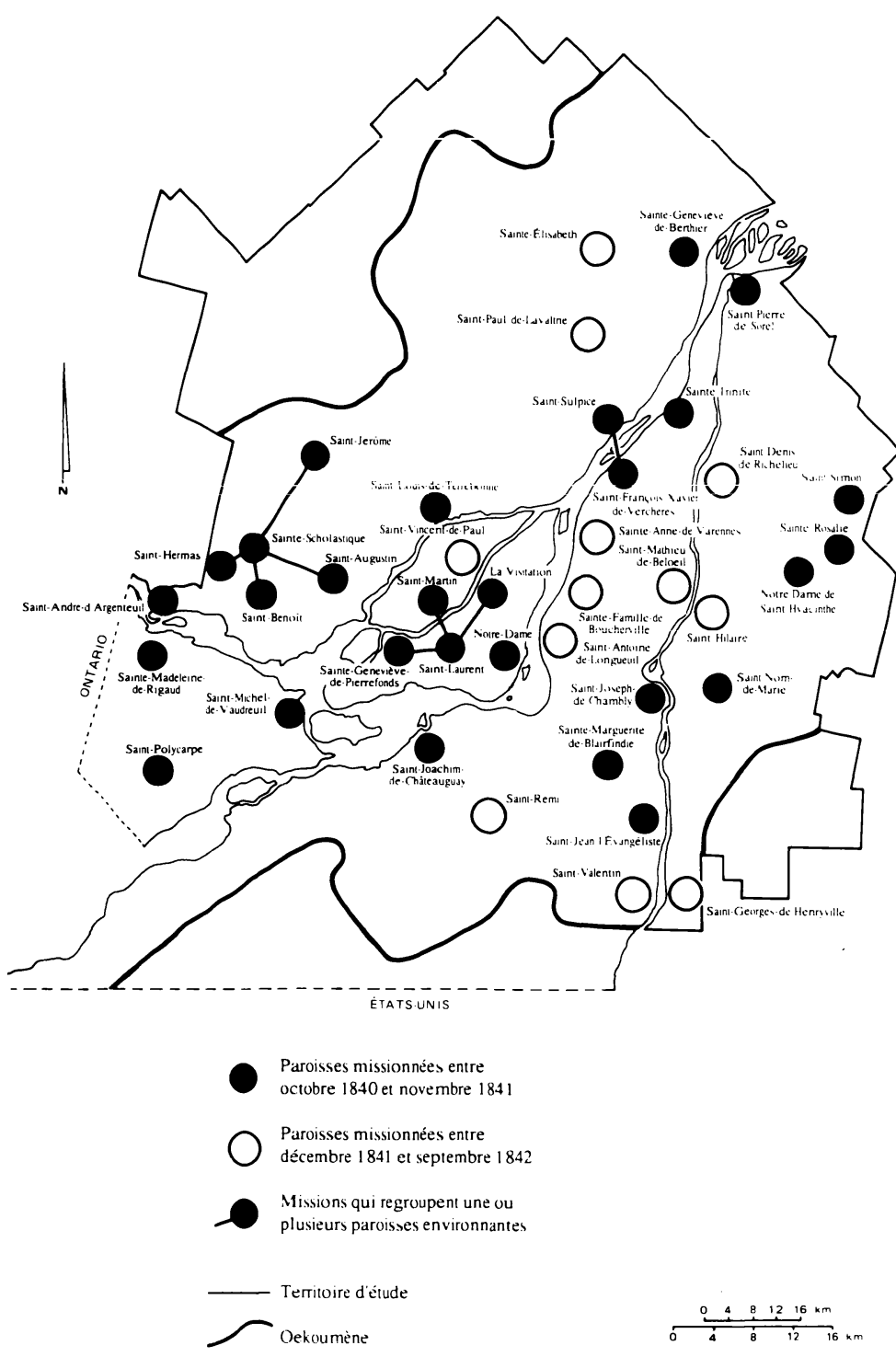

Laboraloire de cartographic. LQAM 
de gros ${ }^{19}$, à la grande déception de Bourget qui espérait la conversion de notables influents. Au curé de Saint-Georges-de-Henryville qui déplore le refroidissement de la ferveur générale un an et demi après une Mission qui avait semblé donner d'excellents fruits, Bourget donne ses encouragements et lui conseille, outre d'instantes prières à la Vierge, de concentrer ses efforts sur la formation aux exercices de piété "des âmes de qui Dieu exige plus de perfection» et de celles qui sont membres de sa congrégation ${ }^{20}$.

En fait on découvrit probablement assez tôt que les transformations effectuées dans l'enthousiasme et la sincérité au cours des Missions populaires avaient peine à se soutenir dans le temps et qu'il fallait élaborer un nouveau programme pastoral pour encadrer les bonnes volontés défaillantes. Ce sera le rôle des sociétés de tempérance et des confréries pieuses dont nous sommes présentement en train d'étudier la croissance sur le territoire montréalais du milieu du XIX ${ }^{\mathrm{e}}$ siècle. Quand aux Retraites, comme l'écrivait d'un ton désabusé le curé de Sainte-Scholastique quatre ans après une de celles qui semblaient avoir connu une belle réussite, "(elles) deviennent une espèce d'amusement. C'est joli, dit-on; les prêtres parlent bien. On pleure quelquefois, on crie, on promet: le spectacle passe, on est bientôt ce qu'on était ${ }^{21}$.

Il serait utile de pouvoir disposer d'instruments de mesure plus objectifs. Aussi avons-nous commencé à examiner différents comportements reliés à l'obligation pascale. Supposant une conduite religieuse assez peu fervente et loin de la pratique unanime, il nous semble vraisemblable que les variations de la ferveur religieuse des masses pourraient être détectées dans les décomptes effectués à chaque année par les curés de l'époque et dont plusieurs ont été conservés. Comme ces premiers travaux ont déjà été publiés ${ }^{22}$, je me contenterai de résumer les résultats les plus significatifs avant d'examiner une nouvelle série qui nous permettra de découvrir l'évolution à long terme des conduites pascales et des attitudes religieuses sous-jacentes.

Nous ne disposons des informations nécessaires à une comparaison des conduites avant et après la Mission que dans le cas de Notre-Dame (Montréal). L'impact, dans ce cas d'une paroisse bien mal en point, est facilement perceptible. Le nombre des communions pascales suivant l'exercice a augmenté de 50 pour-cent. Dans les dix autres cas où nous n'avons de données que postérieures au moment de la retraite, six paroisses signalent

19 Bourget à Mgr l'évêque de Sidyme, Montréal, 7 janvier 1841, ACAM, R.L., 2, p. 285.

20 Bourget à M. Brouillet, Montréal, 27 nov. 1841, ACAM, R.L., 3, p. 257.

21 Bonin à Mgr Bourget, Sainte-Scholastique, 2 mai 1845, ASJCF, dossier SainteScholastique.

22 «La conduite pascale dans la région montréalaise, 1831-1865: un indice des mouvements de la ferveur religieuse", dans R. Litalien, éd., L'Église de Montréal, 1836-1986, Montréal, Fides, 1986, p. 270-284. 
encore des taux de non-participation allant de 31 à 67 pour-cent, ce qui n'est pas très édifiant selon les critères de l'époque. Il se pourrait qu'une explication puisse être trouvée dans la durée plus faible des missions dans certains de ces cas. La transformation des attitudes profondes nécessite autre chose que la présence éclatante et brève d'une vedette, fût-elle Forbin-Janson ou Jean-Paul II.

S'il nous est interdit de conclure à un impact positif instantané partout où se sont tenues des Missions au cours de la campagne de 1840 à 1842 , cela ne nous autorise pas non pius à rejeter l'hypothèse dun réveil reigieux massif amorcé au cours de cette campagne pastorale inédite et qui se serait graduellement implanté à demeure dans les années suivantes. C'est du moins l'interprétation que nous croyons pouvoir donner à la tendance très nette qui apparaît dans l'évolution du taux des absences de communion pascale sur quatre décennies.

Taux de communion pascale dans la région montréalaise, 1836-1881

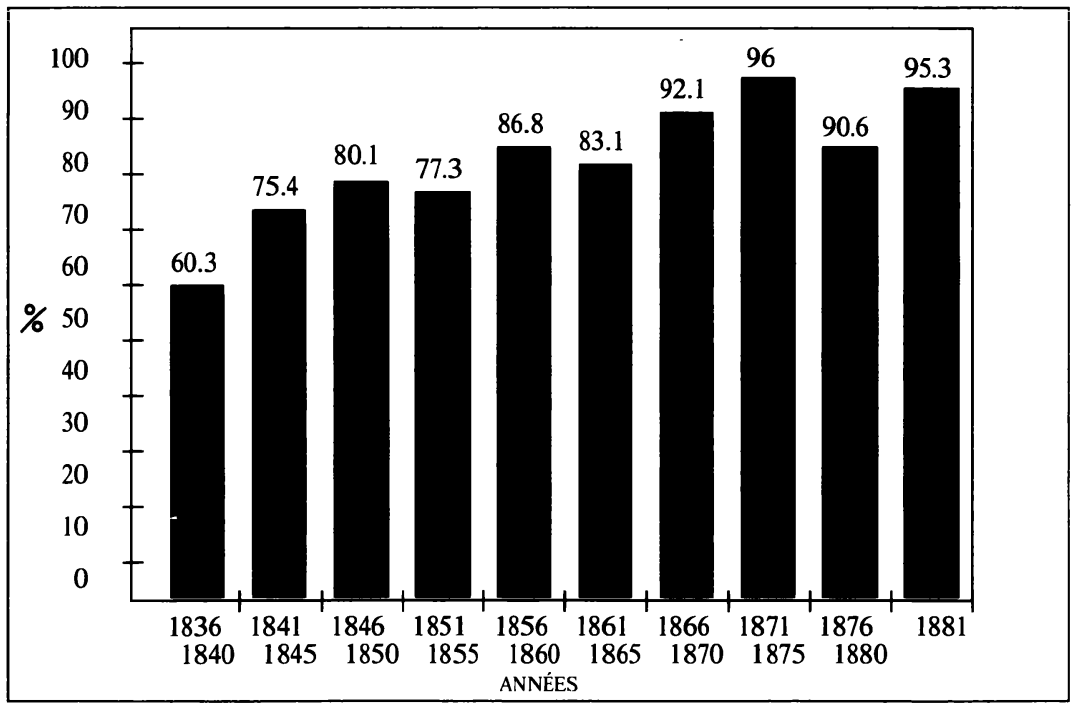

Le choix de l'abstention de communier à Pâques comme indicateur de la ferveur religieuse constitue, comme nous l'avons découvert ${ }^{23}$, une option

23 Louis Rousseau, Vitalité religieuse au XIXe: évaluation de quelques indicateurs, Montréal, UQAM, dép. de sciences religieuses, RRM XIX (Cahiers de recherche-10), juin 1986, polycopié, $10 \mathrm{p}$. 
en faveur d'un critère exigeant. Toujours, à l'époque, les communions sont moins fréquentes que les confessions et les curés eux-mêmes, d'une manière encore inexpliquée, déclarent davantage de pâques faites que de communions pascales, ce qui va contre la logique cartésienne! Si donc nous choisissons de mettre la barre à la hauteur des communions, que constatons-nous?

Il y a une amélioration sensible des choses dès le début des années quarante. En dix ans on compterait une réduction de la déviance pascale de 20 pour-cent. Il pourrait s'agir là du signe le plus manifeste que les Missions, sans tout bouleverser, ont néanmoins donné quelque chose. À partir de ce moment, en effet, s'engage une tendance à l'amélioration graduelle que les fluctuations dues soit à l'état variable de nos données, soit à des effets de conjoncture encore inconnus, ne remettent jamais en cause. Lorsque, à la fin des années soixante, les abstentionnistes passent sous le seuil des 10 pourcent, nous pouvons sans doute affirmer avoir atteint un état d'unanimité religieuse. Un nouvel équilibre socio-culturel s'est implanté et, si nous osons le suggérer pour finir, le processus de revitalisation de la société entrée en crise dans le premier tiers du XIX ${ }^{\mathrm{e}}$ siècle a accompli sa course. À l'abri de sa confession religieuse une Nation-Église est née qui aura la force de vivre un siècle encore. 